\title{
Performance Comparison and Analysis of Power Quality Web Services Based on REST and SOAP
}

\author{
Suxia $\mathrm{Ma}^{1}$, Rui Chen ${ }^{1}$ and Jingjing $\mathrm{Xu}^{1}$ \\ ${ }^{1}$ School of control and Computer engineering, North China Electric Power University, Beijing, China
}

Keywords: Web services; REST; SOAP; Performance comparison; Performance analysis

\begin{abstract}
REST based Web services and SOAP based Web services are two of the most mainstream forms of Web services. The performance of these two services is lack of detailed comparative analysis. In this paper, the power quality application is combined with these two kinds of Web services to realize the sharing of power quality data. Performance tests are performed on power quality Web services interfaces based on REST and SOAP, including stress tests, functional tests, and security tests. The results show that the performance of the REST services is better than that of the SOAP services in particular in real-time response capabilities.
\end{abstract}

\section{Introduction}

Web service technology is a hot topic of computer network technology development in the last ten years. With the increase in the number of Web services, Web services are more widely used, covering every aspect of daily life and work. There are many types of Web services, among which SOAP based Web services and RESTful Web services are two of the most mainstream forms of Web services. SOAP service is inseparable from the SOAP protocol, and the SOAP protocol uses an interactive model based on remote procedure call (RPC), which is better suited to a relatively closed, small application environment [1]. SOAP services use the SOAP message to encapsulate data in envelopes and communicate in XML data formats, and REST services are delivered directly from the HTTP default method through a self-describing method [2]. Therefore, REST services are generally considered to be more concise and better performing. However, the actual operation condition of the two types of services lacks detailed comparison and analysis.

At present, the power system applications began to use power quality basic data services to obtain the required power quality data, to solve the data sharing problem caused by different power quality data format or platform. The application of Web services in power quality monitoring system solves the problem of sharing power quality data due to different data sources and data formats. Because of the huge amount of power quality data, the power quality basic data service has a high demand for response time and returned XML data packets [3, 4]. Choosing the appropriate service architecture technology for power quality Web service has also become an important problem faced by developers.

This paper has developed the power quality basic data Web services based on REST and SOAP. And the performance testing of REST and SOAP Web services is carried out respectively by using the existing Web service testing tools. Through the analysis of the experimental results, it is proved that the performance of REST service is better than SOAP service, and the deployment and operation are better.

\section{Design of power quality Web services}

\subsection{Power Quality Basic Data}

With the construction and development of power quality in China, power enterprises have accumulated a lot of power quality data [5]. The basic data of power quality includes both steadystate data and transient data. The power quality basic data services collects the power quality basic data from different data sources, then preprocesses the data and assembles the data into a specific 
XML format, compresses the XML fragments, and finally provides the functions in the form of Web services Interface [6, 7].

Combine Web services with power quality applications, analyze the data of power quality and publish the results into service, so that users can view the results in the browser. And then we can quickly detect the power quality problem to achieve power quality data sharing.

\subsection{Design of Web Services Interfaces}

According to the IEC 61970 international standard, the data interface specification is as follows: The data exchange interface of the power quality data services is the agreed XML description file, and the Web services adopts the XML data type as the transmission format.

Take the interface "request monitoring point steady-state history data" for example, the data format returned by the interface is shown in Fig. 1.

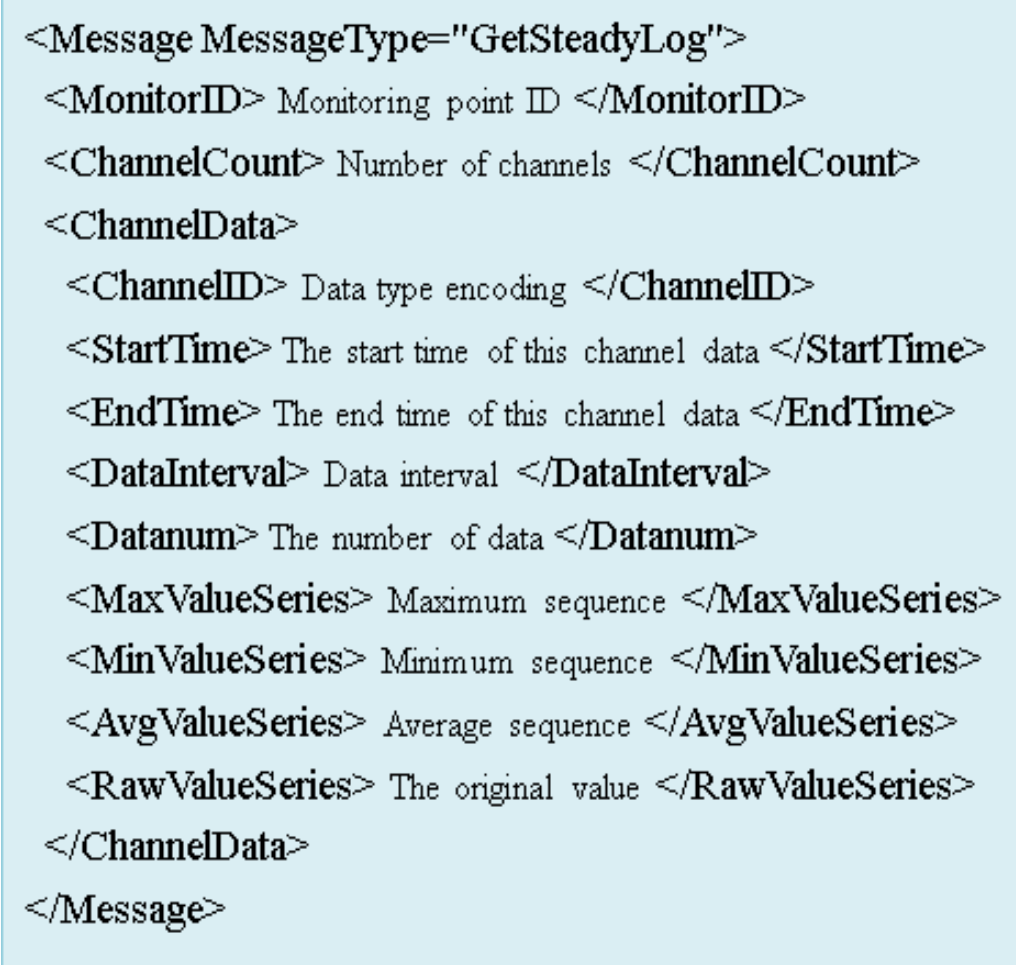

Fig. 1 The data format returned by the interface.

\section{Design of the experiment}

\subsection{Data Sources of the Experiment}

The experimental data are the basic data of power quality, from the basic data platform of power quality. The basic data of power quality should include the following types of data:

- The steady-state historical data of power quality

It refers to the steady-state data collected from the monitoring equipment by specified statistical intervals (such as 1 minute).

- Power quality steady-state real-time data

Contains: voltage / current RMS value, voltage / current spectrum and voltage / current asymmetry component.

- Power quality disturbance event

Contains: power quality transient events and various steady-state parameter overrun events.

- Basic information

Contains: unit information, substation information and monitoring point properties. 


\subsection{Experimental Steps}

Power quality Web services performance test, refers to the call by the power quality data service to measure the response time of Web services, including the response time statistics of multiple users to invoke the Web services concurrently, but also to detect the power quality data service is able to respond to user requests in a timely manner.

There are a lot of testing tools for Web services that can be used to test the connectivity, responsiveness, and performance of Web services. Such as SOAPUI, a cross-platform testing tool, this can support for implementing Web services functionality testing. Others like Jmeter, a Javabased stress testing tool, and SOAPSonar. In this paper, we will test the performance of power quality Web services interfaces based on REST and SOAP, including stress test, functional test and safety test, and compare the test results of the two services.

Considering the time and condition of the network may have an impact on the services call process, this article performs performance tests on REST and SOAP services at different times and in different locations. The number of concurrent users is set to 5, 20, 50, 100 and 500 respectively. The performance of REST and SOAP Web services is tested several times. The results are compared, including the response time, throughput and the returned XML data Package and other aspects are compared.

\section{Experimental results and comparative analysis}

In this paper, the REST and SOAP power quality Web services are executed concurrently with 5, 20, 50, 100, and 500 threads. The time limit is set to 60 seconds, and the interval for each transmission is set to 1 second (1000 milliseconds). Fig. 2 compares the response time for the REST services and the SOAP services.

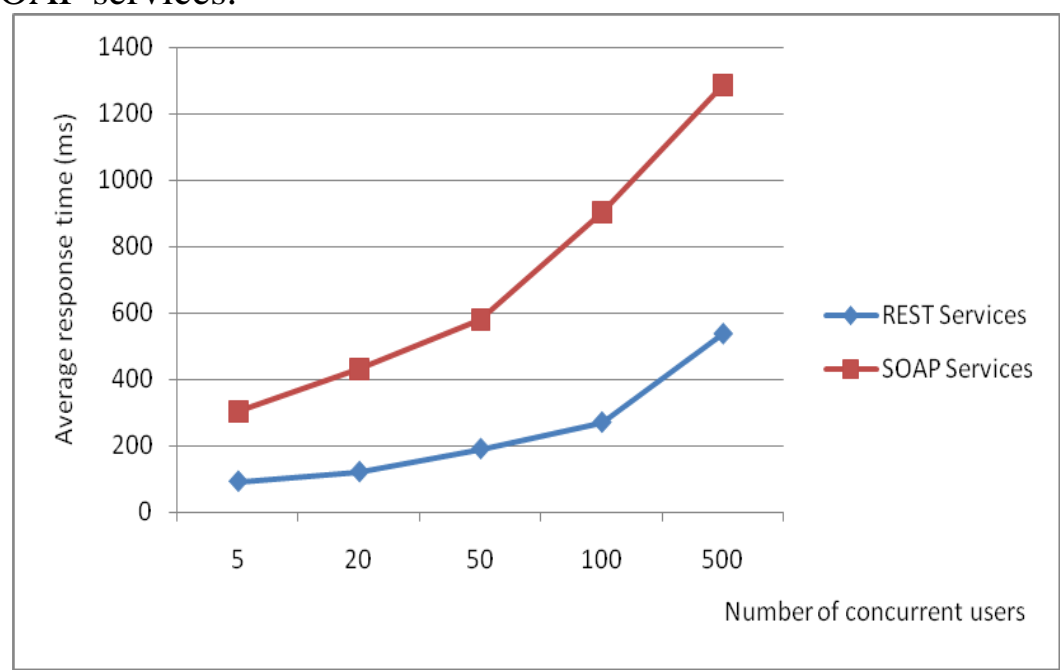

Fig. 2 The response time for the REST services and the SOAP services.

As can be seen from Fig. 2, the average response time of REST services is much smaller than that of SOAP services. With the increase of the number of concurrent users, the gap of response time is gradually increasing. For example, when the number of concurrent users is 5, the average response time of the REST services is only 91.57 milliseconds, the average response time of the SOAP services is 304.07 milliseconds, and the response time of the REST services is much smaller than that of the SOAP services.

Then set the average response time for two Web services. Set the average response time greater than 1 second (1000 milliseconds) is considered failure, and set the maximum response time of Step Maximum 1000 milliseconds. If the time exceeds our set, the test results will be considered failure. Fig. 3 shows the comparison of the number of times the REST service and the SOAP service failed in real time (response time exceeded 1000 milliseconds, which is considered a failure). 


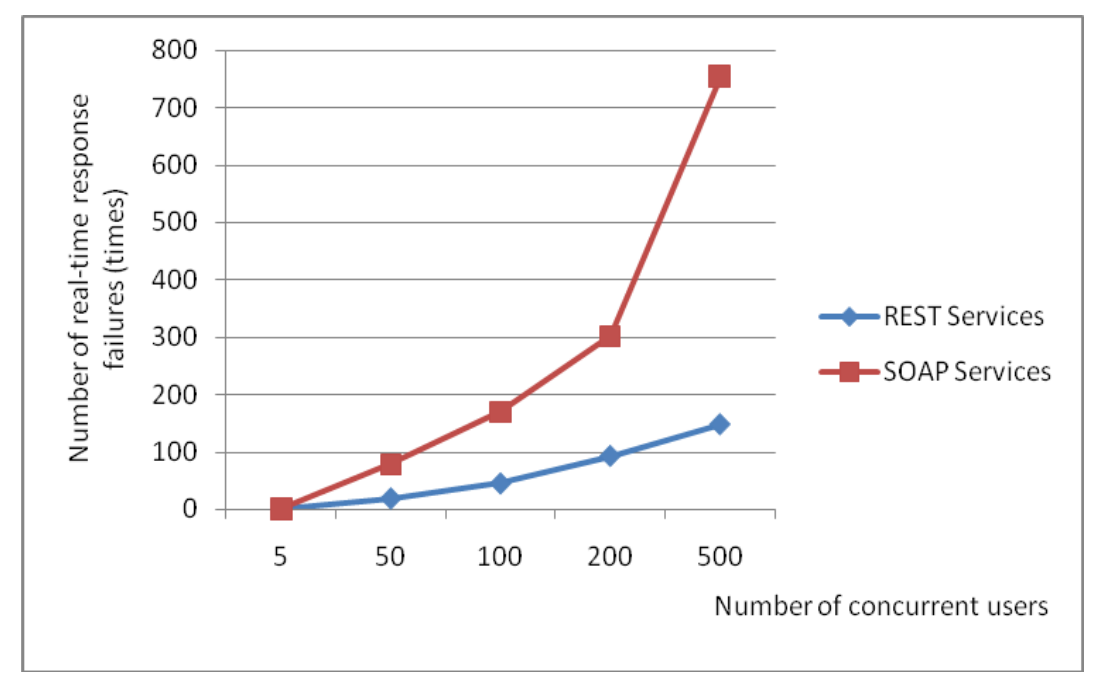

Fig. 3 The number of times the REST service and the SOAP service failed in real time.

As can be seen from Fig. 3, when the number of concurrent users is small (for example, the number of concurrent is 5), the real-time response failures of both services are close to 0 , and the difference is smaller. With the increase of number of concurrent users, especially for the larger number of concurrent, the gap between two services gradually increases, the real-time response failures of SOAP services are much more frequent than REST services. The performance advantage of REST is reflected.

The SOAP services pass data through a SOAP message and needs to parse the data, and REST services can transfer data directly by using HTTP method. REST, in contrast, is superior to the SOAP services in response time, especially in real time.

\section{Conclusion}

Based on the analysis of the basic data of power quality, this paper designed a contrast experiment to compare the REST and SOAP Web services from the aspects of performance. The two kinds of the power quality data services are both called, to measure the response time of the two services, including the response time of multiple users to invoke the Web services concurrently. It also tests whether the power quality Web services is able to respond to users' requests in a timely manner. The results show that the performance of the REST services in response time, throughput, and data transmission is better than that of the SOAP service. And as the number of concurrent users increases, the advantage becomes more and more obvious.

However, due to the limited number and scope of data, the comparison is not comprehensive enough. Further work involves collecting more power quality data and comparing these two types of services on larger data scales and dimensions. The next step is to continue to implement a comparative test of the power quality based on REST and SOAP services from other perspectives, such as the maximum number of concurrent users that the service can afford.

\section{References}

[1]Yang Bo.The Research and Design of Web Service Based on REST Architecture[D]. Zhenjiang. Jiangsu University .2010

[2] GAO Pan-pan, WANG Jian, HUANG Ying, HE Ke-qing. Comparison Analysis of Web Service Based on SOAP and REST on Internet [J]. Small Microcomputer System, 2015, (11): 2417-2421.

[3] ZHAO Xiuping, WU Shuang, MA Suxia. Study on Service Quality Inspection System of Power Quality Basic Data [J]. Power Information and Communication Technology, 2016, (02): 113-118.

[4] Pei Weitao.Study on power quality monitoring technology based on Web service [D]. Baoding: North China Electric Power University, 2009. 
[5] Sun Peng, Ma Suxia.Study on power quality anomaly data detection system based on IEC 61970 standard and Web service [J]. Power Information and Communication Technology, 2015, (06): 8791.

[6] Luan Fujun. Power quality basic data service and detection system [D]. North China Electric Power University, 2014.

[7] Yang Chong. Power quality data service detection system [D]. North China Electric Power University, 2015. 